\title{
Protection des données et assureurs privés: embellie à I'horizon
}

\author{
Hanspeter Kuhn, secrétaire général adjoint \\ Robert Gmür, service juridique de la FMH
}

\section{Deutsch erschienen}

in Nr. 43/2002

\footnotetext{
* Les lois d'assurance sociale prévoient que l'assureur responsabilité civile d'un tiers a un droit de regard sur le dossier dans des domaines délicats de l'assureur social ayant annoncé un droit de recours.
}

Des publications contradictoires telles que les deux précédentes sont utiles pour se positionner et proposer des solutions unilatérales. Dans le domaine de l'assurance-maladie sociale, nous partons de l'idée qu'avec la commission paritaire prévue dans la convention relative aux médecins-conseils des solutions rationnelles pourront être trouvées.

Afin que, dans les autres domaines, on n'en reste pas aux joutes oratoires, une délégation de la FMH s'est assise à la table des négociations avec les représentants de l'Association suisse d'assurances (ASA) à la mi-août 2002. Un collaborateur du préposé fédéral à la protection des données était également de la partie. Il va de soi que les discussions ont d'abord porté sur les questions de principe. Mais, pour certaines questions de détail, des propositions concrètes ont déjà été formulées.

1. Pour commencer, les représentants de l'ASA ont admis un postulat important aux yeux de la FMH, à savoir qu'il était également évident pour l'ASA que l'évaluation critique des conditions médicales à la prise en charge par les assureurs relevait exclusivement du médecin de l'assurance. Si, dans de nombreux cas, une infirmière expérimentée de l'administration des assurances est tout à fait à même d'accorder une garantie de prise en charge des frais, par contre, le refus de cette garantie pour des raisons de non-adéquation (c.-à-d. de violation du principe d'efficacité, d'adéquation et d'économicité) est du ressort exclusif du médecin conseiller.

2. S'agissant de la transparence, dans sa correspondance avec les médecins, l'assureur doit toujours indiquer quelle est sa fonction dans le cas en présence (p.ex. assureur-accidents selon la LAA, assureur indemnités journalières de l'employeur, assureur indemnités journalières du patient avec police individuelle, assureur responsabilité civile d'un tiers auprès duquel l'assureur social ou le patient fait valoir son droit à des dommagesintérêts).
3. En ce qui concerne l'assurance-accidents obligatoire selon la LAA gérée par les assureurs privés, les informations sensibles relatives aux assurés doivent être adressées au médecin conseiller, l'assureur garantit qu'à l'avenir le collaborateur compétent pour les sinistres ne fonctionnera plus comme secrétaire du médecin conseiller. Cette information (p. ex. après ouverture d'une lettre par le secrétariat des médecins conseillers) devra au contraire parvenir directement au médecin conseiller chargé du cas. Une liste commune sera élaborée pour savoir quelles sont les informations considérées comme sensibles pour les assurés. La question de savoir si cette façon de procéder devra concerner uniquement les informations sensibles sans importance pour le sinistre (comme le pense l'ASA) ou bien toutes les informations sensibles n'a pas été tranchée au mois d'août.

4. Dans les recours portant sur des cas de responsabilité de civile, il y a lieu d'éviter les doubles procédures, à savoir que l'assureur responsabilité civile doit consulter d'abord le dossier constitué par l'assureur social (p. ex. les documents de l'assureur accidents qui a pris à sa charge les frais de traitement et de perte de gain après un accident de la circulation*). Ce n'est que lorsque des questions n'ont pas pu être réglées que l'assureur responsabilité civile demande un rapport du médecin traitant. Par exemple pour élucider la prise en charge des frais pour la tenue du ménage, plus ample par l'assurance responsabilité civile que par l'assurance sociale. Si l'assureur responsabilité civile a besoin d'un rapport du médecin traitant, il joindra à sa demande une photocopie de la déclaration de consentement du patient ou indiquera la date de celle-ci. Il faudra voir de ces deux possibilités laquelle sera la plus appropriée, respectivement la plus rationnelle. La date est très importante, car les cas de responsabilité civile s'étendent souvent sur une longue durée et l'état de santé du patient peut s'être modifié dans des domaines délicats depuis la 
déclaration de consentement accordée à l'assureur responsabilité civile; un litige, par exemple, a pu survenir sur la question de savoir si et dans quelle mesure le patient était à nouveau apte au travail.
Tant du côté de la FMH que des assureurs ASA, il conviendra d'examiner ces prochains temps jusqu'où ces premières propositions concrètes sont praticables et quel écho leur est réservé. C'est pourquoi nous serions heureux de connaître les remarques et critiques des praticiens et médecins hospitaliers à cet égard.

Les résultats des consultations internes seront débattus lors des séances. 